\title{
A meso-Triphenylamine-BODIPY Derivative for the Optical Chemosensing of Metal Ions ${ }^{+}$
}

\author{
Raquel C. R. Gonçalves, Sónia C. S. Pinto, Susana P. G. Costa and M. Manuela M. Raposo *
}

Citation: Gonçalves, R.C.R.; Pinto,

S.C.S.; Costa, S.P.G.; Raposo,

M.M.M. meso-TriphenylamineBODIPY Derivative for Optical Chemosensing of Metal Ions. Chem. Proc. 2021, 3, 65. https://doi.org/ 10.3390/ecsoc-24-08291

Academic Editors: Julio A. Seijas and M. Pilar Vázquez-Tato

Published: 14 November 2020

Publisher's Note: MDPI stays neutral with regard to jurisdictional claims in published maps and institutional affiliations.

Copyright: $@ 2020$ by the authors. Licensee MDPI, Basel, Switzerland. This article is an open access article distributed under the terms and conditions of the Creative Commons Attribution (CC BY) license (http://creativecommons.org/licenses/by/4.0/).
Centre of Chemistry, University of Minho, Campus of Gualtar, 4710-057 Braga, Portugal; raquelrainha10@hotmail.com (R.C.R.G.); soniapinto4197@gmail.com (S.C.S.P.); spc@quimica.uminho.pt (S.P.G.C.)

* Correspondence: mfox@quimica.uminho.pt

+ Presented at the 24th International Electronic Conference on Synthetic Organic Chemistry, 15 November-15 December 2020; Available online: https://ecsoc-24.sciforum.net/.

\begin{abstract}
The design and synthesis of organic molecules for recognition of biologically/environmentally important metal ions has emerged as a highly regarded research field. The BODIPY $(4,4-$ difluoro-4-bora-3a,4a-diaza-s-indacene) core is a versatile signaling molecule that can be fine-tuned with functional groups to create selective binding sites to improve its optical properties. As an extension of the work developed in our research group, we report the synthesis and characterization of a BODIPY functionalized with triphenylamino and a formyl group at the meso and 2-position, respectively, for the highly selective detection of $\mathrm{Cu}^{2+}$ and $\mathrm{Fe}^{3+}$. The preliminary study of the BODIPY derivative as optical chemosensor was carried out in acetonitrile solution in the presence of different cations, and interactions with $\mathrm{Cu}^{2+}$ and $\mathrm{Fe}^{3+}$ induced a perceptible color change. UV-visible titrations showed changes in the absorption spectra upon the addition of three equivalents of each cation, with the appearance of a new absorption band at $693 \mathrm{~nm}$.
\end{abstract}

Keywords: BODIPY; metal ions; optical chemosensor; synthesis

\section{Introduction}

Great efforts have been devoted to developing organic molecules as optical chemosensors for application in fields of biomolecular analysis, medical diagnosis, and environmental monitoring. In particular, metal ions have become a prime target, considering that they are essential elements for biological systems but in uncontrolled amounts may represent a critical issue. Classical chemosensors for metal ions contain molecular structures based on polyamines, polyethers, polysulfides, carboxylic acids, hydroxamic acids, and open-chain or macrocyclic structures [1-4].

In the last two decades, BODIPY derivatives have emerged as a novel class of chemosensors for molecular recognition and biological fluorescent labelling. BODIPY shows remarkable optical properties, such as sharp absorption and emission patterns, high molar extinction coefficient of absorbance, high fluorescence quantum yield, and good photostability under physiological conditions [5-7].

The functionalization of the BODIPY framework enables the introduction of highly selective/sensitive binding sites and, simultaneously, modulates its photophysical properties. BODIPYs modified at the meso-position show greater stability than their meso-unsubstituted analogues. In fact, diverse aldehydes can be used to prepare meso-substituted derivatives which allows the design of innovative compounds bearing a large range of functional groups. Furthermore, the BODIPY core is inherently an electron-rich heteroaromatic structure and is therefore very susceptible to regioselective electrophilic aromatic substitution reactions, preferably at positions 2 and $6[8,9]$.

In continuation of our research group's work in the field of chromofluorogenic chemosensors [10-12], we report on the synthesis, characterization, and evaluation of a 
meso-triphenylamine-BODIPY derivative as optical chemosensor of $\mathrm{Cu}^{2+}$ and $\mathrm{Fe}^{3+}$ in acetonitrile solution.

\section{Experimental Section}

\subsection{Methods and Materials}

Nuclear Magnetic Resonance (NMR) spectra were obtained on a Bruker Avance III 400 at an operating frequency of $400 \mathrm{MHz}$, using the solvent peak as an internal reference. The solvents are indicated in parenthesis before the chemical shifts values $(\delta$ relative to tetramethylsilane (TMS). Mass spectrometry analyses were performed at the "C.A.C.T.I.Unidad de Espectrometria de Masas" at the University of Vigo, Spain. All reagents were purchased from Sigma-Aldrich, Acros, and Fluka and used as received. BODIPY precursor 1 was synthesized as previously reported [13]. Thin Layer Chromatography TLC analyses were carried out on $0.25 \mathrm{~mm}$ thick precoated silica plates (Merck Fertigplatten Kieselgel 60F254) and spots were visualized under UV light. Chromatography on silica gel was carried out on Merck Kieselgel (230-400 mesh). UV-visible absorption spectra were obtained using a Shimadzu UV/2501PC spectrophotometer. Fluorescence spectra were collected using a Horiba FluoroMax-4 spectrofluorometer. The relative fluorescence quantum yield was determined by using a $1.0 \times 10^{-5} \mathrm{M}$ solution of rhodamine $6 \mathrm{G}$ in ethanol as standard $\left(\Phi_{\mathrm{F}}=0.95\right)[14,15]$.

\subsection{Synthesis of BODPY Derivative 2}

A mixture of $\mathrm{N}, \mathrm{N}$-dimethylformamide $(23 \mathrm{mmol})$ and $\mathrm{POCl}_{3}(18.2 \mathrm{mmol})$ was stirred for 5 min at $0{ }^{\circ} \mathrm{C}$ under $\mathrm{N}_{2}$ atmosphere. The mixture was allowed to reach room temperature and stirred for an additional $30 \mathrm{~min}$. BODIPY precursor $1(0.127 \mathrm{mmol})$ dissolved in dichloroethane $(7 \mathrm{~mL})$ was added dropwise while stirring. The reaction mixture was heated for $2 \mathrm{~h}$ at $50^{\circ} \mathrm{C}$. After cooling, the solution was poured slowly into $40 \mathrm{~mL}$ of saturated sodium bicarbonate aqueous solution at $0{ }^{\circ} \mathrm{C}$ and stirred during $30 \mathrm{~min}$ at room temperature. Ethyl acetate $(5 \mathrm{~mL})$ was added to the reaction mixture and the resulting organic layer was separated and washed with water $(2 \times 50 \mathrm{~mL})$. The organic layer was dried with anhydrous $\mathrm{MgSO}_{4}$, filtered, and the solvent was evaporated. The crude residue was purified by a silica gel chromatography column, using dichloromethane as eluent. The BODIPY derivative 2 (Figure 1) was obtained as a dark red solid $(6.8 \mathrm{mg}, 15 \%)$.

${ }^{1} \mathrm{H}$ NMR $\left(400 \mathrm{MHz}, \mathrm{CDCl}_{3}\right): \delta=1.66\left(\mathrm{~s}, 3 \mathrm{H}, \mathrm{CH}_{3}-7\right), 1.89\left(\mathrm{~s}, 3 \mathrm{H}, \mathrm{CH}_{3}-1\right), 2.62(\mathrm{~s}, 3 \mathrm{H}$, $\left.\mathrm{CH}_{3}-5\right), 2.83$ (s, 3H, CH3-3), 6.15 (s, 1H, H-6), 7.09-7.21 (m, 10H, Ar-H), 7.30-7.34 (m, 4H, Ar-H), 10.05 (s, 1H, CHO) ppm.

MS (ESI) $m / z(\%): 521\left((\mathrm{M}+2)^{+\bullet}, 35\right), 520\left((\mathrm{M}+1)^{+\bullet}, 100\right), 519\left((\mathrm{M})^{+\bullet}, 34\right), 453(7), 232$ (2); HRMS (ESI) $m / z:(\mathrm{M}+1)^{+\bullet}$ calculated for $\mathrm{C}_{32} \mathrm{H}_{29} \mathrm{BF}_{2} \mathrm{~N}_{3} \mathrm{O}$, 520.2366; found 520.2381.<smiles></smiles>

Figure 1. Structure of BODIPY derivative 2.

\subsection{Chemosensing Study of BODIPY Derivative 2 and UV-Visible Absorption Titrations}

The evaluation of BODIPY derivative $\mathbf{2}$ as an optical chemosensor was carried out in the presence of several cations $\left(\mathrm{Ag}^{+}, \mathrm{K}^{+}, \mathrm{Li}^{+}, \mathrm{Pb}^{2+}, \mathrm{Mn}^{2+}, \mathrm{Cd}^{2+}, \mathrm{Cu}^{2+}, \mathrm{Co}^{2+}, \mathrm{Pd}^{2+}, \mathrm{Ni}^{2+}, \mathrm{Ca}^{2+}\right.$, $\mathrm{Hg}^{2+}, \mathrm{Zn}^{2+}, \mathrm{Fe}^{2+}, \mathrm{Fe}^{3+}$, and $\left.\mathrm{Al}^{3+}\right)$ with environmental and biomedical relevance. Solutions of 
the BODIPY derivative and cations were prepared in acetonitrile at $1.0 \times 10^{-5} \mathrm{M}$ and $1.0 \times$ $10^{-2} \mathrm{M}$, respectively.

A preliminary study was carried out via the addition of 50 equivalents of each cation to the solution of the BODIPY derivative. UV-visible absorption titration experiments were performed by the sequential addition of $\mathrm{Cu}^{2+}$ and $\mathrm{Fe}^{3+}$, as a result of the previous study which revealed selectivity towards these cations. The stability constants were determined from UV-visible spectroscopic titration data, using the HypSpec software [16].

\section{Results and Discussion}

\subsection{Synthesis of BODIPY Derivative 2}

BODIPY precursor 1 was prepared according to a procedure described previously [13]. The synthesis of BODIPY derivative 2 was obtained through the Vilsmeier-Haack formylation of BODIPY precursor 1, meso-substituted with a triphenylamino group, using $N, N$-dimethylformamide (DMF) and phosphorylchloride $\left(\mathrm{POCl}_{3}\right)$ in dichloroethane (Scheme 1). The pure compound was obtained as a dark red solid in 15\% yield.

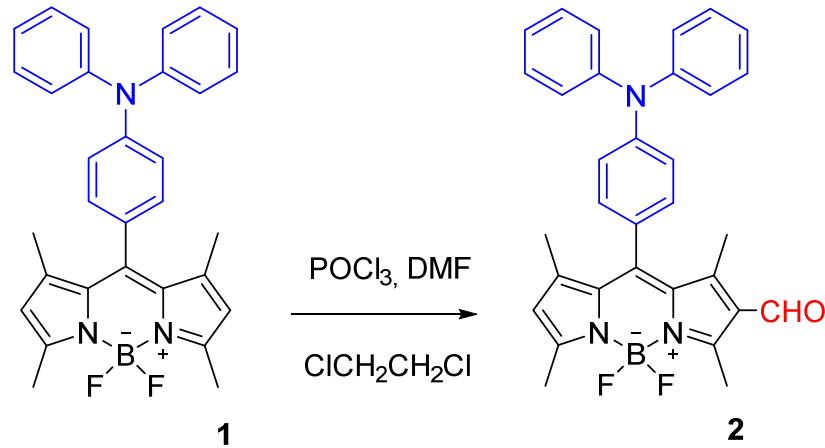

Scheme 1. The synthesis of BODIPY derivative 2 .

The presence of a formyl group at position 2 of the BODIPY core was confirmed by ${ }^{1} \mathrm{H}$ NMR spectroscopy, with the appearance of a singlet at $\delta 10.05 \mathrm{ppm}$. Additionally, the obtained data from mass spectrometry was in agreement with the expected structure.

\subsection{Photophysical Characterization of BODIPY Derivative 2}

The photophysical properties of BODIPY derivative 2 were investigated in acetonitrile solution. The compound showed an intense absorption band $(\log \varepsilon=4.02)$ at $491 \mathrm{~nm}$. Upon excitation at $460 \mathrm{~nm}$, the compound exhibited an emission band at $515 \mathrm{~nm}$. The relative fluorescence quantum yield, determined by using rhodamine 6G in ethanol as standard $\left(\Phi_{F}=0.95\right)$, was found to be low $\left(\Phi_{F}=0.010\right)$. The small quantum yield measured could be ascribed to a carbonyl electron-withdrawing effect exerted by the formyl group linked to the BODIPY core [17].

\subsection{Chemosensing Studies of BODIPY Derivative 2 and Spectrophotometric Titration}

A preliminary evaluation of BODIPY derivative 2 as an optical chemosensor was carried out in the presence of several cations in acetonitrile solution. The chromogenic response of the BODIPY derivative was visible to the naked eye in the presence of $\mathrm{Cu}^{2+}$ and $\mathrm{Fe}^{3+}$. In Figure 2 the color modulation upon addition of 50 equivalents of each cation is observed. As shown, a moderate color change from orange to blue-green exclusively occurred upon the addition of $\mathrm{Cu}^{2+}$ and $\mathrm{Fe}^{3+}$, while other cations were unable to induce a perceptible color change. 


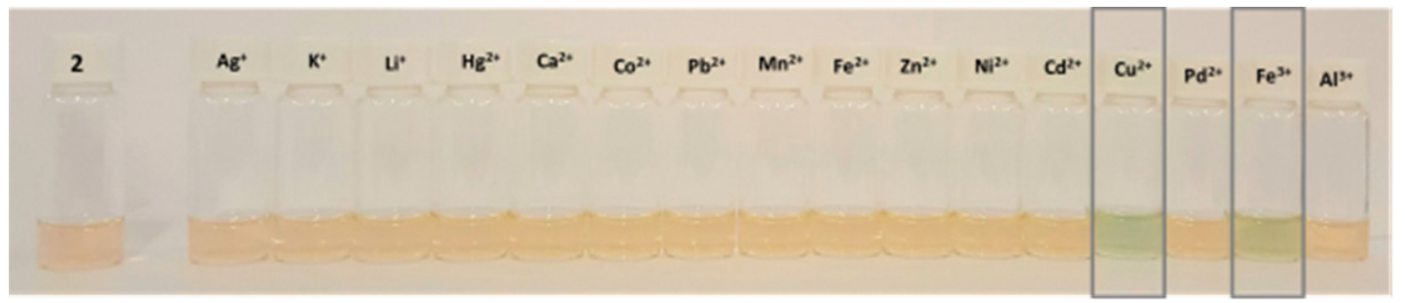

Figure 2. Color changes observed for BODIPY derivative $2\left(1.0 \times 10^{-5} \mathrm{M}\right.$ in acetonitrile $)$ upon the addition of 50 equivalents of different cations.

The UV-visible absorption titration of BODIPY derivative $\mathbf{2}$ was performed in acetonitrile with $\mathrm{Cu}^{2+}$ and $\mathrm{Fe}^{3+}$, as a result of the previous study which revealed a selective chromogenic response towards these cations. As shown in Figure 3, a similar behaviour was observed upon the addition of increasing quantities of $\mathrm{Cu}^{2+}$ and $\mathrm{Fe}^{3+}$. The intensity of the absorption band at $298 \mathrm{~nm}$ decreased, while a new red-shifted band appeared at 693 $\mathrm{nm}$ with a maximum absorbance reached at approximately three equivalents of each cation.
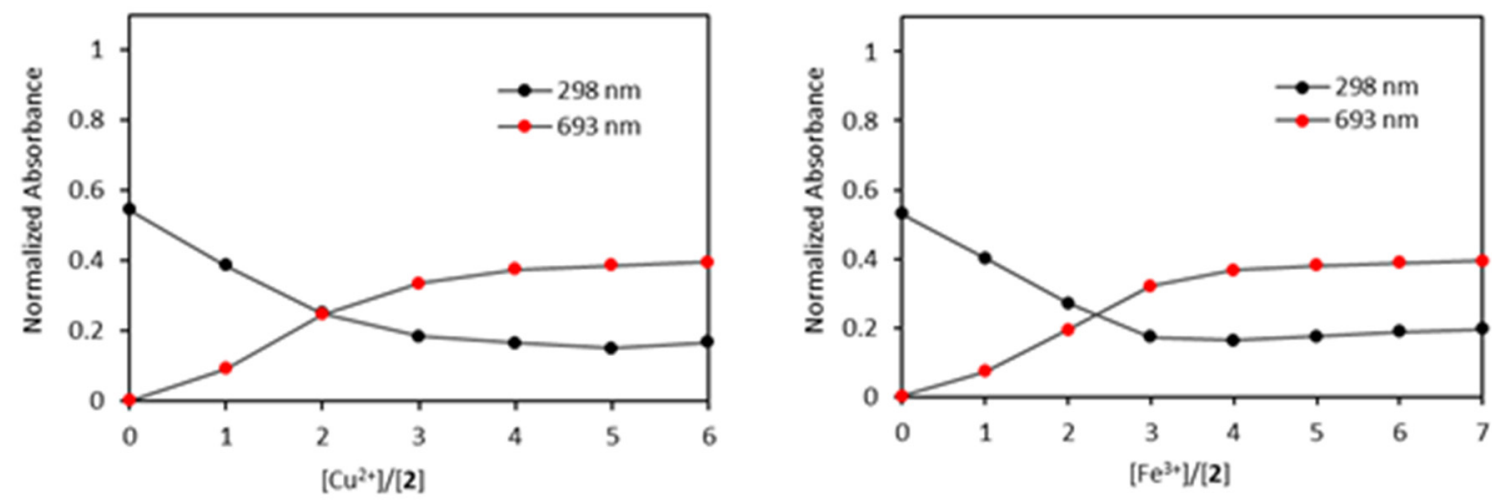

Figure 3. Absorbance at $298 / 693 \mathrm{~nm}$ as a function of the number of equivalents of each cation. Data was obtained from the UV-visible titration spectra of BODIPY derivative $2\left(1.0 \times 10^{-5} \mathrm{M}\right.$ in acetonitrile) upon the addition of increasing quantities of $\mathrm{Cu}^{2+}$ (left) and $\mathrm{Fe}^{3+}$ (right).

Moreover, the interaction between BODIPY derivative 2 with these cations was studied by the determination of the stability constants from the UV-visible spectroscopic titration data, using the HypSpec software. The results suggested the formation of 2:1 cationBODIPY stoichiometry complexes with a logarithm of the stability constant $(\log K)$ of 10.61 for $\mathrm{Cu}^{2+}$ and 10.19 for $\mathrm{Fe}^{3+}$.

\section{Conclusions}

BODIPY derivative $\mathbf{2}$ functionalized with triphenylamino and a formyl group at meso and 2-position, respectively, was synthesized by Vilsmeier-Haack formylation of the BODIPY precursor 1 and its molecular structure was confirmed by ${ }^{1} \mathrm{H}$ NMR and mass spectrometry. The recognition of both $\mathrm{Cu}^{2+}$ and $\mathrm{Fe}^{3+}$ in acetonitrile solution among several cations was observed through a perceptible color change from orange to blue-green. UVvisible titrations showed alterations in the absorption spectra upon the addition of three equivalents of each cation with the appearance of a new absorption band at $693 \mathrm{~nm}$, which clearly indicates that probe 2 can be used for detection of $\mathrm{Cu}^{2+}$ and $\mathrm{Fe}^{3+}$ ions in solution.

Author Contributions: Conceptualization: M.M.M.R.; Experimental work: R.C.R.G., S.C.S.P.; Methodology and formal analysis: S.P.G.C., M.M.M.R.; Writing of original draft: R.C.R.G., S.C.S.P., S.P.C.C., M.M.M.R.; Review and editing: S.P.C.C., M.M.M.R. All authors have read and agreed to the published version of the manuscript. 
Funding: This research was funded by Foundation for Science and Technology (FCT) for financial support to CQ/UM (UID/QUI/00686/2020) and project PTDC/QUI-COL/28052/2017. Thanks are also due to Fundação para a Ciência e Tecnologia (Portugal) for financial support to the Portuguese NMR network (PTNMR, Bruker Avance III 400-Univ. Minho).

Conflicts of Interest: The authors declare no conflicts of interest.

\section{References}

1. Wang, B.; Anslyn, E.V. Chemosensors: Principles, Strategies and Applications, 1st ed.; John Wiley \& Sons: Hoboken, NJ, USA, 2011; Volume 15, pp. 227-295.

2. You, L.; Zha, D.; Anslyn, E.V. Recent Advances in Supramolecular Analytical Chemistry Using Optical Sensing. Chem. Rev. 2015, 115, 7840-7892, doi:10.1021/cr5005524.

3. Kaur, B.; Kaur, N.; Kumar, S. Colorimetric Metal Ion Sensors-A Comprehensive Review of the Years 2011-2016. Coord. Chem. Rev. 2018, 358, 13-69, doi:10.1016/j.ccr.2017.12.002.

4. Formica, M.; Fusi, V.; Giorgi, L.; Micheloni, M. New fluorescent chemosensors for metal ions in solution. Coord. Chem. Rev. 2012, 256, 170-192, doi:10.1016/j.ccr.2011.09.010.

5. Loudet, A.; Burgess, K. BODIPY Dyes and their Derivatives: Syntheses and Spectroscopic Properties. Chem. Rev. 2007, 107, 48914932, doi:10.1021/cr078381n.

6. Boens, N.; Verbelen, B.; Dehaen, W. Postfunctionalization of the BODIPY Core: Synthesis and Spectroscopy. Eur. J. Org. Chem. 2015, 2015, 6577-6595, doi:10.1002/ejoc.201500682.

7. Lu, H.; Shen, Z. BODIPYs and Their Derivatives: The Past, Present and Future; Frontiers Media SA: Lausanne, Switzerland, 2020; doi:10.3389/978-2-88963-786-7.

8. Bañuelos, J.; Arroyo-Córdoba, I.J.; Valois-Escamilla, I.; Alvarez-Hernández, A.; Peña-Cabrera, E.; Hu, R.; Ben, Z.T.; Esnal, I.; Martínez, V.; López Arbeloa, I. Modulation of the Photophysical Properties of BODIPY Dyes by Substitution at Their Meso Position. RSC Adv. 2011, 1, 677, doi:10.1039/c1ra00020a.

9. Zhu, S.; Bi, J.; Vegesna, G.; Zhang, J.; Luo, F.-T.; Valenzano, L.; Liu, H. Functionalization of BODIPY Dyes at 2,6-Positions through Formyl Groups. RSC Adv. 2013, 3, 4793, doi:10.1039/c3ra22610g.

10. Okda, H.E.; El Sayed, S.; Ferreira, R.C.M.; Gonçalves, R.C.R.; Costa, S.P.G.; Raposo, M.M.M.; Martínez-Máñez, R.; Sancenón, F. $\mathrm{N}, \mathrm{N}$-Diphenylanilino-heterocyclic aldehyde-based chemosensors for UV-vis/NIR and fluorescence $\mathrm{Cu}(\mathrm{II}) \mathrm{detection}$. New J. Chem. 2019, 43, 7393-7402, doi:10.1039/c9nj00880b.

11. Presti, M.L.; Martínez-Máñez, R.; Ros-Lis, J.V.; Batista, R.M.F.; Costa, S.P.G.; Raposo, M.M.M., Sancenón, F. A Dual Channel Sulphur-containing Macrocycle Functionalised BODIPY Probe for the Detection of $\mathrm{Hg}(\mathrm{II})$ in Mixed Aqueous Solution. New J. Chem. 2018, 42, 7863-7868, doi:10.1039/c7nj04699e.

12. Esteves, C.I.C.; Ferreira, R.C.M.; Raposo, M.M.M.; Costa, S.P.G. New Fluoroionophores for Metal Cations Based on Benzo[d]Oxazol-5-yl-Alanine Bearing Pyrrole and Imidazole. Dyes Pigments 2018, 151, 211-218, doi:10.1016/j.dyepig.2017.12.040.

13. Gonçalves, R.C.R.; Nogueira, M.B.; Costa, S.P.G.; Raposo, M.M.M. Functionalized BODIPY Derivatives as Potential Fluorescent Labels. Proceedings 2019, 9, 36, doi:10.3390/ecsoc-22-05701.

14. Montalti, M.; Credi, A.; Prodi, L.; Gandolfi, M.T. Handbook of Photochemistry, 3rd ed.; CRC Press: Boca Raton, FL, USA, 2006.

15. Demas, J.N.; Crosby, G.A. Measurement of photoluminescence quantum yields. Rev. J. Phys. Chem. 1971, 75, 991-1024, doi:10.1021/j100678a001.

16. Gans, P.; Sabatini, A.; Vacca, A. Investigation of equilibria in solution. Determination of equilibrium constants with the HYPERQUAD suite of programs. Talanta 1996, 43, 1739-1753, doi:10.1016/0039-9140(96)01958-3.

17. Valeur, B.; Berberan-Santos, M.N. Molecular Fluorescence: Principles and Applications; Wiley-VCH: Weinheim, Germany, 2012. 